\title{
OS EFEITOS DA FADIGA MUSCULAR SOBRE O DÉFICIT DE FORÇA BILATERAL
}

\section{The effects of muscle fatigue on the deficit of bilateral strength \\ Daniel Belloni ${ }^{1}$, Rodrigo Belloni ${ }^{1}$, Alessandro Carielo Albuquerque ${ }^{1}$, Mauro Lúcio Mazini Filho², Estélio Dantas ${ }^{1}$, Vernon Furtado da Silva ${ }^{1}$}

\section{Resumo}

O presente estudo se propôs verificar o efeito da fadiga muscular sobre o déficit de força bilateral (DBL). Participaram deste estudo 21 homens, sedentários, tendo sua perna direita como dominante, apresentando idade média de $24 \pm 0,8$ anos. Antes da aplicação dos testes, todos os participantes realizaram 12 sessões de familiarização. Posteriormente, a força isotônica voluntária máxima (FIVM) foi medida, aleatoriamente, somente da perna direita, somente da perna esquerda e do movimento bilateral. Logo após, os sujeitos foram induzidos a um protocolo de fadiga muscular (PFM). O respectivo PFM incumbia os sujeitos a realizarem cinco a seis séries com somente a perna direita e, imediatamente após, com a perna esquerda, em uma intensidade de $85 \%$ da carga máxima obtida. Um intervalo de 1,5 minutos foi permitido entre cada série. Logo após o PFM, foi realizado o pós-teste para medir a FIVM dos indivíduos, nas mesmas características do pré-teste. O DBL foi calculado nos momentos de pré-teste e pós-teste, para uma análise subseqüente. Dois modelos de ANOVA one-way, concomitantes com o teste post hoc de Tukey, foram usados para analisar os dados resultantes, com nível de significância de $p<0,05$. Após o PFM, os níveis de força dos sujeitos reduziram significativamente $(p<0,05)$, porém não o DBL $(p>0,05)$. Diante destes resultados, é corroborado que o DBL não é influenciado pela fadiga muscular induzida em uma intensidade de $85 \%$ da carga máxima obtida.

Palavras-chave: Déficit Bilateral, Fadiga Muscular, Treinamento.

\section{Abstract}

This study seeks to verify the effect of muscle fatigue on the deficit of bilateral strength (DBL). 21 sedentary men participated in the study, having their right leg dominant, with average age of $24 \pm 0.8$ years. Before the application of the tests, all the participants realized 12 sections of familiarization. Afterwards, the maximum voluntary isotonic force (MVIF) was measured, randomly, only of the right leg, only of the left leg and of the bilateral movement. Shortly after, the subjects were induced to a protocol of muscular fatigue (PMF). The respective PMF assigned the subjects to realizing five to six series with only the right leg and, immediately after, with the left leg, in an intensity of $85 \%$ of the maximum cargo obtained. An interval of 1.5 minutes was permitted between each series. Soon after the PMF, the post-test was realized to measure the MVIF of the individuals, with the same characteristics as the pre-test. The DBL was calculated in the moments of pre-test and post-test, for subsequent analysis. Two models of one-way ANOVA, concomitant with the post hoc test of Tukey, were used to analyse the resultant data, with a level of significance of $p<0.05$. After the PMF, the levels of strength of the subjects reduced significantly $(p<0.05)$, however not the DBL $(p>0.05)$. With these results it is corroborated that the DBL is not influenced by the muscular fatigue induced in an intensity of $85 \%$ of the maximum cargo obtained.

Key words: Bilateral Deficit, Muscular Fatigue, Training.

1. Universidade Castelo Branco - Rio de Janeiro - RJ - Brasil.

2. Universidade Trás do Monte D'Ouros - UTAD - Portugual.

Recebido em 04.09.2007. Aceito em 14.01.2008.

Revista de Educação Física 2008;140:4-12 


\section{INTRODUÇÃO}

A literatura sobre fisiologia neuromuscular descreve várias observações sobre a complexidade do mecanismo neural entre os membros corporais do homem. Um destes fenômenos, bastante conhecido e que data dos anos 60 , recebe a denominação de Déficit de Força Bilateral, ou DBL (Asmussen e Heelboll-Nielsen, 1961). O DBL refere-se a uma diminuição da força muscular máxima, exercida bilateralmente, quando comparada à soma da força muscular máxima, desempenhadas unilateralmente, ou seja, a soma das ações máximas unilaterais suplanta a ação máxima bilateral (Asmussen e Heelboll-Nielsen, 1961; Howard e Enoka, 1991; Oda e Moritane, 1995; Owings e Grabiner, 1998; Owings e Grabiner, 1998; Khodiguian et al., 2003; Kuruganti et al., 2005).

O Déficit de Força Bilateral (DBL) é, normalmente, medido em indivíduos de ambos os gêneros (Schantz et al., 1989), atletas e não atletas (Schantz et al., 1989; Howard e Enoka, 1991), em pequenos músculos, como os das mãos (Li et al., 2001; Li et al., 2001; Taniguchi et al., 2001) e, principalmente, em grandes músculos de membros inferiores e superiores (Khodiguian et al., 2003; Kuruganti et al., 2005; Gabriel et al., 2006; Behm et al., 2003).

Geralmente, quando tal fenômeno biológico é encontrado, alcança elevados percentuais, variando entre 3 a $25 \%$ da força total (Owings e Grabiner, 1998; Vandervoort et al., 1984).

Não existe DBL quando músculos ou grupos musculares heterogêneos são ativados simultaneamente, como, por exemplo, na ativação simultânea da musculatura extensora do joelho e flexora do cotovelo (Schantz et al., 1989; Howard e Enoka, 1991). Muitas vezes, quando tais músculos ou grupamentos musculares heterogêneos são acionados juntos, ocorre uma facilitação do membro avaliado (Schantz et al., 1989; Howard e Enoka, 1991; Behm et al., 2003).

Verificando-se a robustez das evidências que comprovam o fenômeno do DBL, poder-se-ia pensar em um paradoxo a existência de evidências contrárias. Todavia, elas existem, acenando a possibilidade de que o mesmo não é, de fato, tão concreto assim. Renomados pesquisadores, ao investigarem o DBL, relataram, em alguns casos, inexistência do mesmo (Howard e Enoka, 1991; Häkkinen et al., 1995; Häkkinen et al., 1996; Häkkinen et al.,1997; Jakobi e Cafarelli, 1998). Deste modo, não foi identificada a existência de diferenças significativas entre a força unilateral somada e a bilateral. Ainda, em alguns casos, um considerável número de pesquisadores constatou uma facilitação de força bilateral (FBL) em suas experimentações (Schantz et al., 1989; Howard e Enoka, 1991; Behm et al., 2003; Khodiguian et al., 2003), revelando que, em parâmetros de comparação, a força unilateral somada, exercida separadamente, era menor que a força bilateral desempenhada conjunta.

De qualquer maneira, a busca pela identificação do mecanismo, ou ainda, mecanismos responsáveis pela ocorrência do DBL, tem fomentado uma variedade de hipóteses, nas quais se incluem as noções de que esse fenômeno seria causado por falta de treinamento (Howard e Enoka, 1991), por interferência entre os hemisférios cerebrais (Oda e Moritane, 1995; Li et al., 2001; Li et al., 2001; Taniguchi et al., 2001) e por seletiva inibição de unidades motoras (Serche, 1975; Vandervoort et al., 1984; Vandervoort et al., 1987; Owings e Grabiner, 1998; Owings e Grabiner, 1998). Contudo, a última possibilidade citada não tem recebido muita atenção, embora, quando investigada, surjam relatos contraditórios que, de certa forma, associam o DBL a uma restrição de unidades motoras do tipo 1 (UMsT1) ou unidades motoras do tipo 2 (UMsT2).

Uma outra forma de investigação, nesta linha, mais precisamente a que explora a utilização de recursos farmacológicos, tem indicado que o DBL é causado por uma contribuição diminuída de UMsT1 (Serche, 1975), visto que testes fisiológicos, contraproducentemente, têm revelado que o DBL é, mais provavelmente, ocasionado por uma falha na ativação das UMsT2 (Vandervoort et al., 1984; Vandervoort et al., 1987). Alguns autores, em seus estudos (Vandervoort et al., 1984; Vandervoort et al., 1987), revelaram uma possível restrição das UMsT2 como causa do DBL. Mas, em aporte a tal constatação, outros autores mostram que essa possibilidade é completamente infundada (Owings e Grabiner, 1998; Owings e Grabiner, 1998).

A compreensão acurada sobre o mecanismo subjacente motivador do fenômeno do DBL é de imprescindível importância para qualquer indivíduo que esteja envolvido com atividades relacionadas à reabilitação, bem como com a preparação física no treinamento esportivo. Por exemplo: no esporte, quando a ação inibitória 
TABELA 1

CARACTERÍSTICAS DOS VOLUNTÁRIOS EM TERMOS DE PESO, DE ALTURA E DE IDADE.

\begin{tabular}{lcccc}
\hline & $\mathbf{N}$ & IDADE (Anos) & PESO (kg) & ALTURA (cm) \\
\hline Voluntários & 21 & $24 \pm 0,8$ & $70,5 \pm 4,6$ & $175 \pm 3,8$ \\
\hline
\end{tabular}

Os valores são apresentados em médias e desvio padrão da média; $N$ = 21 .

das contrações bilaterais de músculos homólogos, causada pelo fenômeno do DBL, diferentemente da ação de apenas um membro, provocaram uma redução da ativação das unidades motoras de ambos os membros, Howard e Enoka (1991) encontraram a presença do DBL associada com o tipo de atividade esportiva, cujo rendimento demonstrava ser maior. Este déficit de força, embora pequeno, era de total significância para diferentes atividades. Portanto, a ação do DBL, para alguns esportes, possui uma repercussão significativamente importante, pois a utilização de um só membro, como é o caso do tênis, do beisebol (arremessador) e do dardo olímpico, tende a maximizar a performance nesses esportes.

Outro componente do acervo de mecanismos fisiológicos humanos que tem recebido atenção, nos últimos anos, é a fadiga muscular. De fato, este fenômeno encontra-se atuante durante qualquer atividade esportiva, principalmente quando tal atividade começar a ultrapassar os limites do funcionamento de determinadas estruturas fisiológicas (Oda e Moritane, 1995; Owings e Grabiner, 1998). Conseqüentemente, o mecanismo de fadiga muscular age implícito, como uma defesa de possíveis detrimentos fisiológicos causados por níveis excedentes de atividades.

Sabe-se que a fadiga muscular interfere intimamente nos níveis de produção de força e de coordenação hábil-motriz em atividades de qualquer natureza. Contudo, o que não se conhece, ainda, é se este mecanismo possui alguma relação com outros mecanismos subjacentes, como, por exemplo, o DBL, podendo, assim, prejudicar a performance atlética, ou, talvez, retardar qualquer iniciativa de terapias voltadas ao equilíbrio de forças uni e bilateral.

Como foi delineado, fortes e compreensíveis dúvidas pairam sobre a real natureza do mecanismo subjacente responsável pela ocorrência do DBL. Assim sendo, considerando-se a importância desta questão científica, o presente estudo teve o objetivo de verificar os efeitos da fadiga muscular sobre o DBL.

\section{METODOLOGIA}

\section{Amostra}

Após um chamado amplo, 21 indivíduos, do gênero masculino, sedentários, com idade média de $24 \pm 0,8$ anos, com nenhum histórico de lesão ortopédica ocorrida em seu membro inferior, ofereceram-se, voluntariamente, para participar da pesquisa. Todos indicaram sua perna direita como membro dominante, o que foi posteriormente evidenciado, através de uma sistemática preferência destra ao chutar uma bola de futebol. Os participantes eram aparentemente saudáveis, sem qualquer experiência em exercícios resistidos, sendo que nenhum deles estava envolvido, naquele momento, em algum tipo de programa de exercícios físicos regulares, desde um ano até a data de admissão à pesquisa. Os dados antropométricos dos avaliados encontram-se plotados na TABELA 1.

Os dados de participação, ou não participação, foram investigados através de um questionário próprio. Foi adotada, ainda, com o objetivo de inclusão/exclusão amostral, a possibilidade dos participantes portarem patologias neurológicas em qualquer grau, bem como outras que pudessem estar associadas a funções músculo-esqueléticas e/ou cardiovasculares, que pudessem interferir no desempenho das tarefas inerentes aos testes e ao programa definido da pesquisa.

Para participarem do estudo, todos os indivíduos responderam negativamente aos itens do questionário PAR-Q e foram informados dos procedimentos metodológicos da pesquisa, incluindo os possíveis desconfortos, riscos e benefícios do estudo, antes de assinarem o termo de consentimento, de acordo com as normas da resolução 196/96, do Conselho Nacional de Saúde sobre pesquisa envolvendo seres humanos. 


\section{Instrumentos}

Um equipamento de Leg press 45 (Marca Physicus, modelo 2001) foi usado para medir a força isotônica concêntrica voluntária máxima (FICVM) no movimento de pressão de pernas. Em ambos os experimentos e durante todos os exercícios de aprendizado motor realizados, os sujeitos foram posicionados sobre o banco do equipamento em uma posição intimamente similar. A principal finalidade deste procedimento era a de buscar a máxima semelhança na execução do movimento inter e intra-avaliados, durante todo o período de familiarização e testes realizados, bem como o de manter o conforto e a confiabilidade de que todos os avaliados realizassem o movimento de pressão de pernas com o mesmo grau de esforço.

\section{Familiarização ao exercício}

Antes de se aplicar o protocolo de testes inerentes à pesquisa, os participantes visitaram o laboratório durante 12 vezes consecutivas, com intervalo mínimo de 48 horas e máximo de 72 horas entre as respectivas visitas, tendo, esta situação, o objetivo de fazer os participantes atenderem a um protocolo de exercício de familiarização, incluindo oito sets de 12 repetições no aparelho, utilizando-se, no treinamento, um parâmetro de intensidade do mesmo nível que a escala de Borg (10 - 12). O principal propósito deste procedimento foi o de promover a familiarização dos participantes com o exercício padrão, tornando possível a diminuição de possíveis erros no desempenho motor dos testados. Deste modo, instruções padronizadas sobre a técnica correta de execução do exercício foram passadas aos participantes.

Todos os sujeitos deste estudo realizaram as sessões de familiarização ao treinamento com repetições na mesma cadência. Para que isso ocorresse, utilizou-se um metrônomo profissional (Boss DB 30, 2006) para auxiliar os participantes. Foram gastos um segundo no trabalho concêntrico e três segundos na ação excêntrica. Cada um dos bips produzidos pelo metrônomo durava um segundo. Conseqüentemente, todos se adaptaram e foram treinados a executar as repetições na mesma velocidade e nas mesmas condições.

Com relação à posição adotada pelos avaliados, o avaliador manteve-se atento durante todo o protocolo de familiarização, pois qualquer variação no posicionamento das articulações envolvidas, poderia ativar outros músculos e, efetivamente, prejudicar o efeito do aprendizado motor da fase de familiarização.

\section{Medida de força muscular por meio de levantamento de peso}

O teste de uma repetição máxima (1RM), conhecido, mundialmente, como teste de carga máxima, usando o protocolo de De Lorme e Watkins (1945), incumbia cada participante a realizar uma execução máxima, na maior velocidade possível, até a obtenção da carga máxima. A cada nova tentativa, realizavam-se incrementos progressivos na carga do aparelho, sendo permitido repousos de cinco minutos entre as tentativas. Foram concedidas quatro tentativas para a obtenção da carga máxima. Caso a mesma não fosse obtida após as referidas tentativas, o avaliado era convidado a comparecer a uma sessão extra para finalização dos procedimentos para se obter o valor real da carga máxima. O objetivo do teste de carga máxima era obter a maior carga possível no movimento de pressão de pernas, até o momento em que o avaliado não conseguisse mais realizar o levantamento completo e de forma correta. Dessa forma, validou-se, como carga máxima, a maior carga levantada pelos avaliados de maneira correta.

As cargas máximas, somente da perna direita (SPD), somente da perna esquerda (SPE) e do movimento bilateral (MBL), foram aleatoriamente obtidas de cada um dos participantes. Após obter e computar a carga máxima gerada SPD, SPE e do MBL, no pré-teste (pré), foi permitido um longo intervalo e, logo após, aplicado o pós-teste, em condições análogas ao pré-teste. Precedentemente aos pós-testes desempenhados, foi realizado o protocolo de fadiga muscular (PFM).

\section{Protocolo de fadiga muscular}

O respectivo protocolo, para induzir os sujeitos à fadiga muscular, consistia em realizar os sets de fadiga na subseqüente ordem. Primeiro, realizava-se uma série com a perna direita e, imediatamente, outra série com a perna esquerda. Depois de efetuar as séries, com ambas as pernas, inicialmente com a direita e, subseqüentemente, com a perna esquerda, era permitido um repouso de 1,5 minutos. Na TABELA 2, pode-se observar o protocolo de fadiga muscular.

Uma característica comum no PFM foi a execução do movimento de extensão/flexão do joelho na mesma 
TABELA 2

PROTOCOLO DE FADIGA MUSCULAR.

\begin{tabular}{lccc}
\hline $\mathbf{1 R M} \%$ & Séries & Repetições & Recuperação (min') \\
\hline 85 & $5-6$ & 6 & $01: 30$ \\
\hline
\end{tabular}

cadência. Todos os avaliados desempenhavam a fase concêntrica e excêntrica do movimento, em um tempo total de quatro segundos, com o auxílio de um metrônomo. Durante a execução de cada repetição, era gasto um segundo na fase concêntrica e três segundos na fase excêntrica, totalmente análoga ao treinamento realizado no período da familiarização. Conforme já especificado anteriormente, cada um dos bips produzidos pelo metrônomo durava um segundo.

Um estímulo motivacional verbal foi, insistentemente, fornecido durante todos os testes inerentes à pesquisa, em número de vezes igual para todos os participantes.

\section{Tratamento estatístico}

Os dados obtidos, durante as várias fases do estudo, foram tratados através de análises estatísticas de natureza descritiva e inferencial. Na modalidade descritiva, as médias e os desvios-padrão da média do grupo, nas diversas etapas (pré-teste e pós-teste), foram a referência de importância às análises. No estilo inferencial, foram utilizados instrumentos paramétricos, tendo-se a análise de variância como o principal modelo. Deste modo, para se analisar os dados resultantes, associados aos testes de fadiga nas ocasiões de pré-teste e pós-teste, utilizou-se uma análise de variância de medidas repetidas (ANOVA One-way), modelo 2 (momentos) x 1 (carga máxima obtida), que comparou os dois momentos de testes do grupo. Uma segunda ANOVA, do mesmo tipo, foi realizada com o objetivo de se verificar a possibilidade da existência de diferença significativa entre os DBL obtidos pelo grupo, durante os momentos de teste. Em todas as ANOVA foi usado, concomitantemente, o teste post hoc de Tukey para, se fosse o caso, identificar a procedência da diferença encontrada. Um nível de probabilidade de $\alpha<0,05$ foi usado para todas as análises, como valor significativo aceitável, indicando o ponto das diferenças reveladas.

\section{RESULTADOS}

Analisando-se os efeitos do protocolo de fadiga muscular (PFM), nos níveis de força produzidos pelos indivíduos, observa-se que, embora a força não tenha diminuído como o esperado, essa diferença se mostrou estatisticamente significativa (TABELA 3).

O déficit de força bilateral (DBL) dos sujeitos, nos estágios de pré-teste para o pós-teste, subseqüente ao protocolo de fadiga muscular, não apontaram nenhuma diferença significativa entre eles. Pode-se observar, no GRÁFICO 1, que estes dados são bastante similares.

\section{DISCUSSÃO}

Como descrito na literatura científica, o fenômeno do Déficit de Força Bilateral pode ser pensado como uma leve diminuição da ativação neural no recrutamento de unidades motoras, quanto ao desenvolvimento de uma ação muscular máxima exercida bilateralmente, comparativamente à soma das ações musculares máximas

\section{TABELA 3}

LEVANTAMENTOS MÁXIMOS EM KILOGRAMAS (KG) NO EQUIPAMENTO, EM PERÍODOS ANTERIORES E POSTERIORES AO PROTOCOLO DE FADIGA.

\begin{tabular}{lccc}
\hline & \multicolumn{3}{c}{ FADIGA MUSCULAR } \\
\cline { 2 - 4 } MOMENTOS AVALIADOS & SPD $(\mathbf{k g})$ & SPE $(\mathbf{k g})$ & MBL $\mathbf{( k g})$ \\
\hline Pré-teste & $180 \pm 21$ & $181 \pm 23$ & $346 \pm 42$ \\
Pós-teste & $156 \pm 20^{*}$ & $156 \pm 22^{*}$ & $299 \pm 36^{*}$ \\
\hline
\end{tabular}




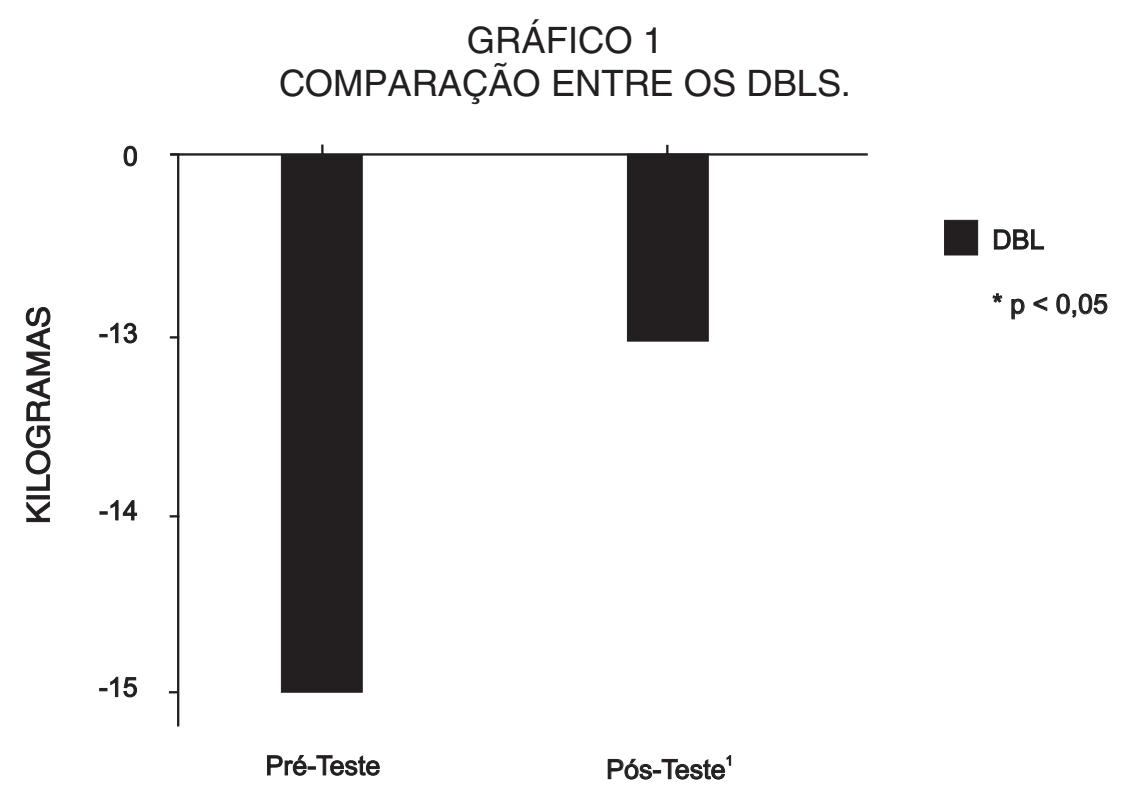

*Diferença revelada estatisticamente entre os DBLs durante os momentos de teste

desempenhadas em condição unilateral (Kuruganti et al., 2005). Ao refletir sobre o fenômeno do DBL, fortes e compreensíveis dúvidas pairam sobre a verdadeira natureza do mecanismo subjacente, responsável pela ocorrência deste fenômeno, e, implicitamente, sobre a possibilidade de ter, o mesmo, uma relação direta com restrições seletivas associadas às funções de determinadas unidades motoras. Em decorrência desta carência científica, este estudo investigou, mais especificadamente, a real possibilidade da magnitude de um comprovado DBL poder ser alterado, de certa forma, através da aplicação de um treinamento de força, protocolado em função de estados de fadiga muscular de baixa e/ou alta intensidade.

O racional que subsidia o esboço deste estudo parte do princípio de que, se, de fato, a ocorrência de um caso de DBL pode ser explicado, de maneira inerente a restrições seletivas de determinadas unidades motoras, o mesmo tenderia a ocorrer somente em uma situação em que tal seletividade pudesse ser exercida, ou seja, sem condição de fadiga muscular. Por outro lado, o mesmo não deveria ocorrer caso os níveis de fadiga interferissem elevadamente na produção de força de determinadas unidades motoras, revertendo, conseqüentemente, tal ocorrência fenomenológica. O fato de vários autores, como Howard e Enoka (1991), Owings e Grabiner (1998), Owings e Grabiner (1998), Taniguchi et al.(2001), Schantz et al. (1989), não aceitarem esta concepção, desperta a curiosidade sobre uma explicação alternativa e/ou, até mesmo, de comprovação de uma ou outra linha de pensamento.

Ao analisar os resultados referentes ao comportamento da força muscular e do déficit bilateral, subseqüentes ao protocolo de fadiga muscular, induzido em alta e baixa intensidade, promove suporte muito mais para a dúvida do que para o conhecimento mais concebido. Isto porque os testes inerentes aos dois níveis de intensidade, aqui manipulados, evidenciaram que, mesmo considerando-se uma relação isolada dos níveis de força correspondentemente ao DBL, em momentos de pré e pós-teste, as diferenças entre eles não se mostraram estatisticamente significativas. Isto se explica devido ao fato de que a fadiga muscular, embora tenha influenciado significativamente os níveis de força isoladamente, quando comparada, em termos dos DBL, não tenha ocorrido.

Embora a dimensão do DBL pareça não ser associação com a restrição de certas unidades motoras, tem sido sugerido que a força voluntária, quando exercida acima de aproximadamente $80 \%$ de sua máxima competência, devido à sua dependência de determinados fatores neurogênicos, em indivíduos não treinados e pouco treinados, pode ser um fator preponderante para a maioria das dificuldades oriundas dos trabalhos desempenhados bilateralmente (Sale, 1998; Kamen, 2004; Gabriel et al., 2006). Conseqüentemente, partindo deste princípio teórico, o sistema nervoso central (SNC) destes sujeitos possui 
um baixo status de capacidade para sustentar a ativação das unidades motoras tipo 2 (UMsT2), visto que seu limiar de excitabilidade é significativamente elevado. Portanto, esta respectiva situação poderia, de alguma maneira, colaborar para a decorrência de um DBL. Por outro lado, as respostas fisiológicas deste fenômeno, decorrentes à fadiga muscular, não forneceram sustentação clara para a presunção de que este fenômeno poderia ser causado por alguma restrição seletiva de unidades motoras do tipo 1 e/ou 2. Este fato se produziu mesmo com a aparente presença da fadiga muscular. Contudo, tal hipótese especulada sobre uma possível restrição seletiva de unidades motoras do tipo 1 e/ou 2, durante ações desempenhadas bilateralmente, pode não ser completamente correta, devido ao instrumento de avaliação (fadiga) não ser um bom preditor para comprovação científica deste fato.

Os resultados referentes à presente pesquisa, desta forma, não se podendo, sob uma perspectiva científica, identificar relação entre força produzida sob estado de fadiga diferenciada, DBL e seletiva restrição de unidades motoras, rejeitam a idéia de que o referido déficit possa ser uma função atribuída diretamente ao SNC, como comprovado, cientificamente, em estudos prévios por outras linhas de pesquisa (Howard e Enoka, 1991; Oda e Moritane, 1995; Jakobi e Cafarelli, 1998; Behm et al., 2003; Khodiguian et al., 2003). Portanto, não sendo esta a única explicação do fenômeno sob estudo, que outro mecanismo poderia ser pensado como motivador do mesmo?

Alguns autores, normalmente, estabelecem uma relação entre DBL e o seguimento motor do SNC alto, envolvidos na ação de força uni ou bilateral (Howard e Enoka, 1991; Oda e Moritane, 1995; Taniguchi, 2001). Esta concepção reflete, ainda, a possibilidade de que o DBL possa ter uma dependência dos mecanismos reflexos reguladores da conduta motora humana, em ações simétricas e assimétricas (Khodiguian et al., 2003). Parte da explicação desta hipótese pode ser análoga a mecanismos que regulam a função muscular, principalmente em grupos contra-laterais (Behm et al., 2003). Veja-se, por exemplo, o reflexo extensor cruzado mediado por ações análogas cruzadas, desempenhado em situação de deambulação, marcha e/ou corrida de qualquer natureza. A pergunta, que aqui cabe, seria a de se a contralateralidade dos mecanismos reflexos, nestas ações, poderiam influenciar tempos e cargas de contração nos músculos análogos, mantendo, com isto, a força normal em cada segmento corporal (ex.: perna direita e perna esquerda), mas limitando a força conjunta dos membros comparados, em função da diminuição somatória e correspondente aumento por segmento isolado. Esta idéia, embora possível, quando analisada à luz da neurologia, carece de aplicação científica sob regime de pesquisa que a possa comprovar.

Em termos de resultados oriundos da pesquisa ora em discussão, o máximo que se pode afirmar, ainda assim dentro dos limites da metodologia utilizada, seria de que o respectivo protocolo de fadiga muscular não provocou alterações significativas no DBL dos sujeitos designados para este estudo.

\section{CONCLUSÃO}

Em síntese, esta análise teve concreta incumbência de estender concebíveis conhecimentos sobre os efeitos da fadiga muscular sobre o Déficit de Força Bilateral (DBL). Surpreendentemente, os resultados, aqui constatados, encontravam-se bastante diferentes do esperado, principalmente devido ao estudo proposto, nesta ocasião, tangenciar-se com pesquisas previamente publicadas. Conseqüentemente, a questão geral que motiva a real natureza do fenômeno estudado, sob perspectiva de restrições seletivas de determinadas unidades motoras, permanece aberta para discussões futuras.

Em conclusão, fica evidente que o fenômeno DBL parece não ser influenciado pela fadiga muscular induzida pelo treinamento de força, mas, sim, por possíveis mecanismos centrais do sistema somático, como já comprovado por outras linhas de pesquisa.

\section{REFERÊNCIAS BIBLIOGRÁFICAS}

ASMUSSEN E, HEELBOLL-NIELSEN K. Isometric muscle strength of adult men and women. Comm Dan Natl Assoc for Infant Paralysis $1961 ; 2: 3-43$. 
BEHM DG, POWER KE, DRINKWATER EJ. Muscle activation is enhanced with mult- and uni-articular bilateral versus unilateral contractions. Can J Appl Physiol 2003; 28(1):38-52.

DE LORME TL, WATKINS AL. Techniques of progressive resistance exercise. Arch Phys Med 1948; 29 (5):263-73.

GABRIEL DA, KAMEN G, FROST G. Neural Adaptations to Resistive Exercise: Mechanisms and Recommendations for Training Practices. Sports Med 2006; 36(2):133-49.

HÄKKINEN K, PASTINEN UM, KARSIKAS R, LINNAMO V. Neuromuscular performance in voluntary bilateral and unilateral contraction and during electrical stimulation in men at different ages. Eur J Appl Physiol 1995; 70: 518-27.

HÄKKINEN K, KRAEMER WJ, KALLINEN M. Bilateral and unilateral neuromuscular function and muscle cross-sectional area in middle-aged and elderly men and women. J Gerontol A Biol Sci Med Sci 1996; 51:21-9.

HÄKKINEN K, KRAEMER WJ, NEWTON RU. Muscle activation and force production during bilateral and unilateral concentric and isometric contractions of the knee extensors in men and women at different ages. Electromyogr Clin Neurophysiol 1997; 37: 131-42.

HOWARD JD, ENOKA RM. Maximum bilateral contractions are modified by neurally mediated interlimb effects. J Appl Physiol 1991; 70(1): 306-16.

JAKOBI JM, CAFARELLI E. Neuromuscular drive and force production are not altered during bilateral contractions. J Appl Physiol 1998; 84: 200-6.

KAMEN G. Neural issues in the control of muscular strength. Res Q Exerc \& Sport 2004;75: 3-08.

KHODIGUIAN N, CORNWELL A, LARES E, DICAPRIO PA, HAWKINS A. Expression of the bilateral deficit during reflexively evoked contractions. J Appl Physiol 2003; 94: 171-8.

KURUGANTI U, PARKER P, RICKARDS J, TINGLEY M, SEXSMITH J. Bilateral isokinetic training reduces the bilateral leg strength deficit for both old and young adults. Eur J Appl Physiol 2005; 94:175-9.

LI Z-M, ZATSIORSKY VM, LI S, DANION F, LATASH ML. Bilateral multifinger deficits in symmetric key-pressing tasks. Exp Brain Res 2001; 140:86-94.

LI S, DANION F, LATASH ML, LI Z-M, ZATSIORSKY VM. Bilateral deficit and symmetry in finger force production during two-hand multifinger tasks. Exp Brain Res 2001; 141:530-40.

ODA S, MORITANE, T. Cross-correlation of bilateral differences in fatigue during sustained maximal voluntary contraction. Eur J Appl Physiol 1995; 70:305-10.

OWINGS TM, GRABINER MD. Fatigue effects on the bilateral deficit are speed dependent. Med Sci Sport Exec 1998; 30(8):1257-62.

OWINGS TM, GRABINER MD. Normally aging older adults demonstrate the bilateral deficit during ramp and hold contractions. J Gerontol Biol Sci 1998; 53(6)425-29.

SALE DG. Neural Adaptations to resistance training. Med Sci Sports Exerc 1998; 20:135-45.

SCHANTZ PG, MORITANE T, KARSON E, JOHANSSON E, LUNDH A. Maximal voluntary force of bilateral and unilateral leg extension. Acta Physiol Scand 1989; 136:185-92.

SERCHE NH. Isometric rowing strength of experienced and inexperienced oarsmen. Med Sci Sports 1975; 7:280-3.

TANIGUCHI Y, BORÍS B, FRANCK V, BORNNT M. Deficit in motor cortical activity for simultaneous bimanual responses.

Exp Brain Res 2001; 137:259-68. 
VANDERVOORT AA, SALE DG, MOROZ J. Comparison of motor unit activation during unilateral and bilateral leg extension. J Appl Physiol 1984; 56:46-51.

VANDERVOORT AA, SALE DG, MOROZ J. Strength-velocity relationship and fatigability of unilateral and bilateral leg extention. Eur J Appl Physiol 1987; 56:201-5.

\section{Endereço para Correspondência:}

Daniel Teixeira Belloni Avenida Ministro José Fabrino Baião, 304 - Thomé

Cataguases - MG - Brasil CEP: $36774-184$

Tel.: (32) 8846-6332 e-mail: dtbelloni@yahoo.com.br

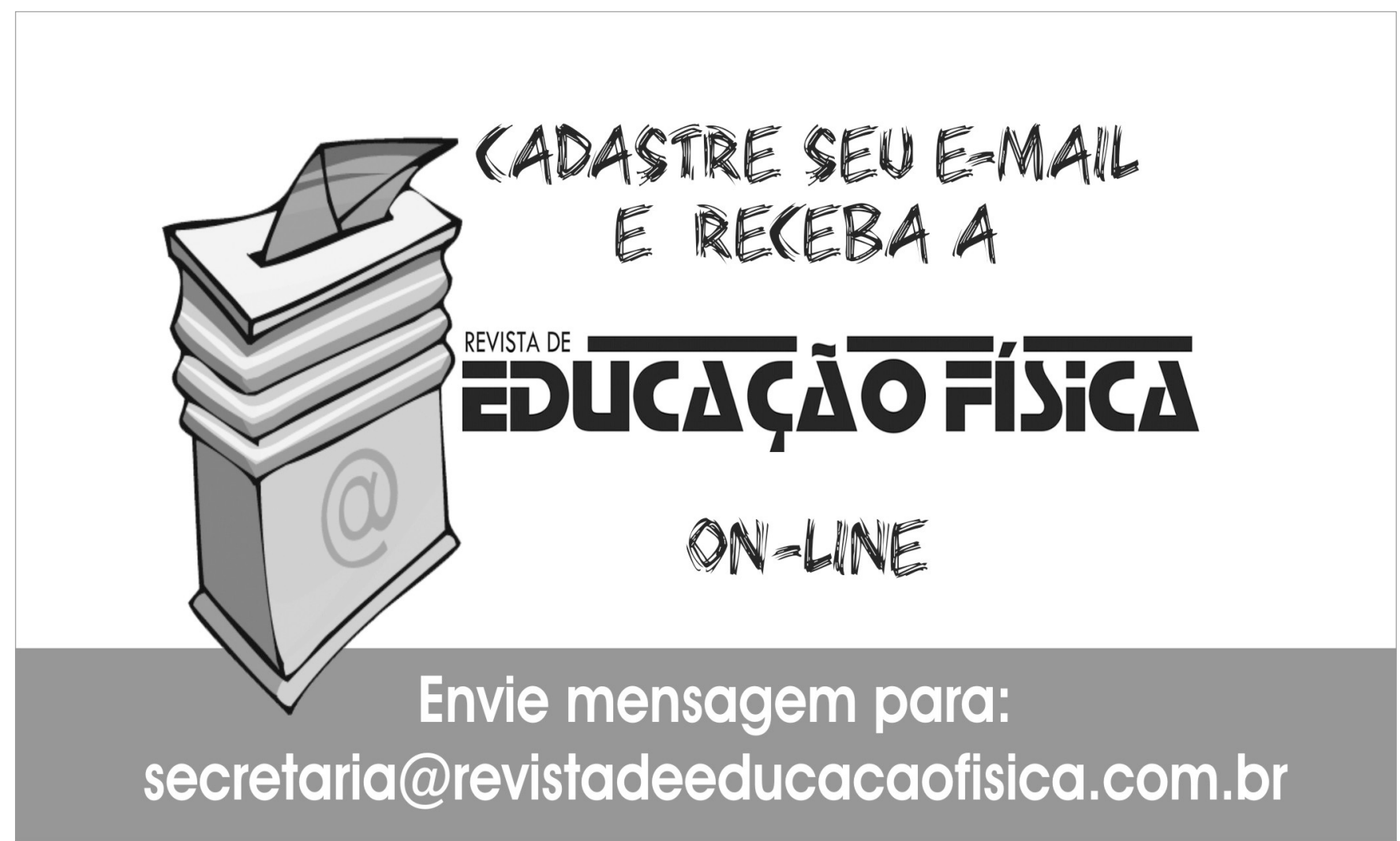

